Original Article

\title{
Determination of the fascicle length of the gastrocnemius muscle during calf raise exercise using ultrasonography
}

\author{
Shintarou Kudo, MSc, RPT ${ }^{1,2)^{*}}$, Tomoyuki Hisada, RPT ${ }^{2)}$, Takanori Sato, RPT ${ }^{3)}$ \\ 1) Department of Physical Therapy, Morinomiya University of Medical Sciences: 1-26-16 Nanko-kita, \\ Suminoe ward, Osaka city 559-0034, Japan \\ 2) Gradutate School of Health Science, Suzuka University of Medical Science, Japan \\ 3) Department of Physical Therapy, International Institute of Medical Therapy, Japan
}

\begin{abstract}
Purpose] The purpose of this study was to find a strength training protocol which maintains isometric contraction of the triceps surae during dorsal flexion of the ankle. [Subjects] The left feet of 22 young normal volunteers who did not have orthopedic injuries or lower limb pain participated in this study. [Methods] All subjects performed four sets of five repetitions of four sets calf-raise (CR) exercise at were (1) $60 \mathrm{bpm}$ without a pedestal, (2) $60 \mathrm{bpm}$ with a pedestal, (3) $90 \mathrm{bpm}$ without a pedestal, and (4) $90 \mathrm{bpm}$ with a pedestal. The fascicle length of the lateral head of the gastrocnemius and ankle angle were measured using ultrasonography and a video camera. The CR exercise was divided into two or three phases using the kinematics of the ankle. The average change in fascicle length over the five repetitions of each phase were compared. [Results] The change of the fascicle length during the hyper-dorsiflexion phase was significantly smaller than during the other two phases. [Conclusion] It is possible that eccentric CR exercises have progressed to motor learning of the isometric contraction during counter movement, and improved the release of elastic energy of the Achilles tendon during running, jumping, and other athletic activities.

Key words: Gastrocnemius, Isometric contraction, Eccentric calf raise exercise
\end{abstract}

(This article was submitted Aug. 4, 2015, and was accepted Sep. 17, 2015)

\section{INTRODUCTION}

In the leg, the triceps surae muscle group comprises the soleus muscle and the medial and lateral heads of the gastrocnemius. The lateral head of the gastrocnemius (GL) originates from the lateral epicondyle of the femur, and the medial head of the gastrocnemius (GM) originates from the medial epicondyle of the femur. The soleus muscle originates from the posterior aspect of the femoral head, the proximal quarter of the shaft of the fibula a, the tibia's soleal line, and the middle third of its medial border, and the fibrous arch between the tibia and fibula. These muscles insert on a common tendon known as the Achilles tendon ${ }^{1)}$, which is the largest and strongest human tendon. Neptune et al., have demonstrated that the soleus primarily generates forward angular momentum, while the gastrocnemius generates backward angular momentum ${ }^{2}$. The difference between the soleus and the gastrocnemius is in their roles in the generation of horizontal and vertical ground reaction forces ${ }^{2)}$. The

*Corresponding author. Shintarou Kudo (E-mail: kudo@ morinomiya-u.ac.jp)

C2015 The Society of Physical Therapy Science. Published by IPEC Inc. This is an open-access article distributed under the terms of the Creative Commons Attribution Non-Commercial No Derivatives (by-ncnd) License $<$ http://creativecommons.org/licenses/by-nc-nd/3.0/>. triceps surae therefore plays two roles: first, it contributes to greater plantar flexion torque and stabilizes the ankle; and second, it allows the rolling forward of the total mass of the foot, lower leg, and body during the stance phase of the gait. The stance phase has been divided into five phases: the initial contact, loading response, mid stance, terminal stance, and pre-swing ${ }^{3)}$. From the mid stance to the terminal stance, the largest ankle plantar flexion moment is required to raise the center of the body mass, and the ankle rocker and the forefoot rocker function as forward progression mechanisms during walking. These functions are needed for strong activities of the triceps surae muscles. The standing heel raise, called the "calf raise" (CR), is a commonly prescribed exercise for improving the strength and power of the ankle plantar flexors ${ }^{3)}$.

Training to strengthen the muscles of the triceps surae is therefore clinically important. According to Perry, the ability to perform at least 30 calf raises by in single leg is required to make the muscles strong enough for normal walking ${ }^{4}$.

Recently, motor learning theory was applied to gait practice in a clinical setting. Continuous skills were retained nearly perfectly over long intervals. However, discrete skills showed marked performance losses during the same retention intervals ${ }^{4}$. There is extensive evidence in support of body weight-supported treadmill training for gait practice after impairments such as stroke, femoral proximal fracture, and osteoarthritis. However, in almost all clinical cases, some 
discrete skill practices, aimed at improving gait ability, are performed in expectation of the transfer of learning before continuous skill practices, such as body weight-supported treadmill training. Transfer of learning is usually defined as the gain (or loss) in the capability of performance in one task as a result of practice or experience of some other task. In the transfer of learning, the degree of the transfer depends on the similarity between the two tasks, and the transfer depends on the number of "identical elements" that exist in common between two tasks ${ }^{5)}$. However, there was no evidence of the most effective elements for transfer of learning are not known.

Ultrasonography is widely used by the physical therapists in both clinical setting and laboratory settings, and many of studies have used to be investigated muscle morphology such as the muscle thickness, width, and area in static condition $^{6-8)}$. The dynamics of the triceps surae are known to exhibit isometric contractions during motions such as walking, jumping, and running ${ }^{9,10)}$. Fukunaga et al., reported that in the late stance phase of walking, the triceps surae muscles perform isometric contractions ${ }^{9}$. Kawakami et al., reported that the gastrocnemius performs the isometric contraction during dorsiflexion of the ankle, and a large proportion of the power originates from elastic energy when the tendon is extended ${ }^{10)}$. The fascicles maintain a near-constant length when the muscle is active. The tendon of the muscle stretches during the major part of the stance phase and recoils in pushoff. Both these mechanisms favor locomotor economy ${ }^{9)}$. The strength of the triceps surae is important for human bipedal locomotion because it is needed to raise the center of body mass through plantar flexion of the ankle. Strength training of the triceps surae to improve motion capability is necessary to maintain the muscle group's isometric contraction during dorsal flexion of the ankle. These muscle contractions are related through their properties of viscosity and elasticity. The purpose of this study was to find a strength training protocol which maintained isometric contraction of the triceps surae during dorsal flexion of the ankle.

\section{SUBJECTS AND METHODS}

The left feet of 22 healthy normal volunteers (mean age $22.9 \pm 5.1$ years) who did not have orthopedic injuries or lower limb pain, and who provided their informed consent, participated in this study. All the subjects performed four sets of five repetitions of CR exercise with alterations in the two conditions: rhythm and starting position. The rhythm of the calf-raise was either 60 or 90 beats per minute (bpm), and was controlled by a digital metronome. The starting position was either normal standing or standing with the forefoot on a $6 \mathrm{~cm}$ pedestal with dorsal flexion of the ankle. Therefore, the four sets were performed at (1) $60 \mathrm{bpm}$ without a pedestal, (2) $60 \mathrm{bpm}$ with a pedestal, (3) $90 \mathrm{bpm}$ without a pedestal, and (4) $90 \mathrm{bpm}$ with a pedestal. These exercises were performed these in a random order. This study was approved by the ethics committee of Morinomiya University (2014-078).

Reflective markers ten millimeters in diameter were mounted over the lateral side of the knee, the lateral malleolus, and the fifth metatarsal head. All CRs were recorded using a digital video camera in the sagittal plane. The recordings were translated from .avi file format to .jpeg using the free software program, Aviutl. The ankle angle, which was the right angle made by the three markers, was measured using the free image processing software program, Image-J (National Institutes of Health, Washington, DC, USA). The GL muscle fascicle lengths during the CRs were recorded using B-mode real-time ultrasonography (My Lab 25, Esaote Corporation), which was synchronized with the digital video camera. Ultrasonography and digital video camera were both recorded with $30 \mathrm{~Hz}$. A linear ultrasound probe $(12 \mathrm{MHz})$ was fixed using fixation devices at both the thickest part of the lower leg and the center of the GL muscle, and the longitudinal axis of the GL muscle was recorded. The pennation angle (the right angle between the fascicle and deep aponeurosis of the GL muscle) and the thickness of the GL muscle (the distance from the superficial fascia to the deep aponeurosis) were measured using Image-J. A single examiner blinded to the condition of the CR exercises measured the fascicle length. This method has high reliability (intraclass correlation [ICC] $=0.91$ ), as confirmed in a pilot study. The fascicle length was calculated using the equation [1]:

Fascicle length $(l)=a \cdot \cos ^{-1} \theta, \theta$ : pennation angle...[1]

The CR exercise was divided into two or three phases using the kinematics of the ankle. The first phase was defined as the elevation from the starting position to the peak plantar flexion angle. The second phase was defined as the down phase from the peak plantar flexion angle to the neutral zero position, and the third phase was defined as the hyper-dorsiflexion phase from the neutral zero position to the maximum dorsi-flexion position. The third phase was only present when the pedestal was used.

The excursion of the ankle, peak dorsiflexion angle, plantar flexion angle, and angular velocity during each CR were calculated. After five repetitions, measurements of each of the three parameters were averaged and the four conditions were compared using one-way ANOVA with Bonferroni's correction. Moreover, the change in of the fascicle length during each CR was calculated. The average change in fascicle length over the five repetitions in each phase was compared. If the phase which was less of fascicle length changing were shown, using two-way ANOVA with Bonferroni's correction. Significance was accepted for values of $\mathrm{p}$ $<5 \%$.

\section{RESULTS}

Both the excursion and angular velocity of the ankle showed no significant differences among the four conditions. The peak ankle dorsiflexion was significantly different between eccentric CR and normal CR (Table 1). The change in the fascicle length during the hyper-dorsiflexion phase was significantly smaller than in the other two phases (Fig. 1), and the change in the fascicle length was not significantly different between the $60 \mathrm{bpm}$ and $90 \mathrm{bpm}$ (Table 2). 
Table 1. The ankle kinematics difference among CR conditions

\begin{tabular}{lcccc}
\hline & \multicolumn{2}{c}{ With pedestal } & \multicolumn{2}{c}{ Without pedestal } \\
\hline & $60 \mathrm{bpm}$ & $90 \mathrm{bpm}$ & $60 \mathrm{bpm}$ & $90 \mathrm{bpm}$ \\
\hline Angle excursion (degrees) & $42.9 \pm 10.6^{*}$ & $38.3 \pm 11.5$ & $35.5 \pm 8.3^{*}$ & $37.6 \pm 8.5$ \\
Angular velosity (degrees/sec.) & $105.0 \pm 23.1^{*}$ & $125.6 \pm 35.2^{*}$ & $102.9 \pm 35.4^{\dagger}$ & $123.0 \pm 30.4^{\dagger}$ \\
Maximum dorsi-flex angle (degrees) & $19.2 \pm 10.0^{*}$ & $14.9 \pm 11.8^{*}$ & $1.1 \pm 2.8^{*}$ & $0.1 \pm 2.5^{*}$ \\
\hline
\end{tabular}

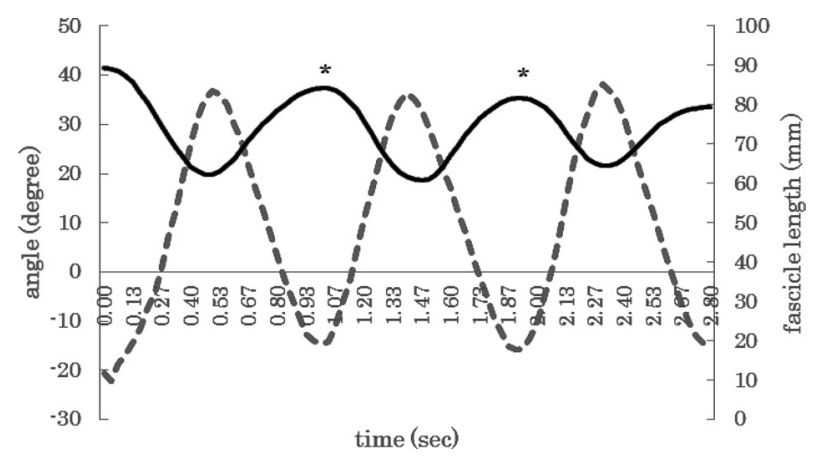

Fig. 1. Graph of the changing fascicle length during $60 \mathrm{bpm}$ calf raises with a pedestal

Solid line; fascicle length. Dashed line; angle of the ankle. The fascicle length showed a little change, even the angle of the ankle showed significant changes $\left(^{*}\right)$.

\section{DISCUSSION}

The skeletal muscles have two different properties: viscosity and elasticity. Viscosity is known to be in proportional to the velocity of materials, and elasticity is known to be in proportional to the magnitude of displacement. The dynamics of the skeletal muscles during motion affects these properties. Therefore, it was our hypothesis that at the time of isometric contraction, when the fascicle length was increased, the viscosity and elasticity of the skeletal muscles would also be increased.

In this study, four sets of the CR exercise were performed. Changes in the frequencies of the CR exercises led to changes in the angular velocity, and the change in the starting position led to change in the excursions of the ankle. The results of the angular velocity and the excursion were significantly different between the starting conditions with and without the pedestal at both frequencies of exercise repetition. Moreover, the peak dorsiflexion angle was significantly different between the two pedestal conditions. Thus, Participants exhibited higher muscle elasticity when CRs were performed. CRs at the $90 \mathrm{bpm}$ repetitions showed higher muscle viscosity than at $60 \mathrm{bpm}$ conditions. The CR with pedestal conditions additionally had a as hyper dorsi-flexion phase. During the hyper dorsi-flexion phase, the change in fascicle length was smaller than in the other phases. This indicates that the typical contraction of the GL during the hyper dorsi-flexion phase was very similar to that of an isometric contraction, while the typical contraction during the elevation phase and drop phase were concentric
Table 2. Changing fascicle length difference among three phases

\begin{tabular}{lrrl}
\hline & Phase 1 & Phase 2 & Phase 3 \\
\hline $60 \mathrm{bpm}$ & $26.6 \pm 11.5$ & $20.9 \pm 8.7$ & $4.0 \pm 3.6^{*}$ \\
$90 \mathrm{bpm}$ & $29.7 \pm 12.7$ & $18.6 \pm 8.7$ & $8.1 \pm 3.9$ \\
\hline
\end{tabular}

and eccentric, respectively. Kawakami, et al., described that the gastrocnemius in plantar-flexion as movement with counter-movement on a sliding board which worked almost isometrically, and that this work was performed through exercise performance by relying on the tendon to storing and releasing elastic energy ${ }^{10)}$. Sugisaki, et al., showed that as the intensity of the rebound action at the ankle increased in a drop jump, the tendinosus tissues were further stretched and provided greater speed when shortened, thereby enhancing muscle and tendon unit positive power ${ }^{11)}$. Moreover, Kawakami reported that subjects learned to effectively use this muscle-tendon interaction for greater work generation by modulating the activation strategy of muscle fibers through practice $^{12}$. Therefore, it is our conclusion that the exercise that showed similar with isometric contractions with dorsiflexion of the ankle should be performed by elderly people and athletes.

Eccentric CR exercise is known to improve the strength of the calf muscle and has an evidence base as a conservative treatment for medial tibial stress syndrome ${ }^{13)}$. The eccentric exercise remains a popular conservative Achilles tendinopathy treatment technique ${ }^{14-16)}$. It is possible that eccentric CR exercises have progressed to motor learning of isometric contraction during the counter movement, and improved the release of elastic energy from the Achilles tendon during running, jumping, and other athletic activities.

This study had an important limitation. The fascicle length was measured at one specific site. It is possible that typical muscle contraction is not the same throughout the muscles. Thus, while the midpoints of the muscle belly may showed isometric contraction, the proximal sites of the muscle belly might not always show isometric contraction. Nonetheless, isometric contraction of the GL is very important. Therefore, it was the revelation to observe that the performance of eccentric CR exercise can elicit isometric contractions during the hyper dorsi-flexion phase is important. In future studies, the effect of eccentric $\mathrm{CF}$ exercises on athletic performance during activities such as running and jumping should be investigated. 


\section{REFERENCES}

1) Williams PL, Warwick R: Gray's anatomy. London: Churchill livingstone,1989, pp 647-648.

2) Neptune RR, McGowan CP: Muscle contributions to whole-body sagittal plane angular momentum during walking. J Biomech, 2011, 44: 6-12. [Medline] [CrossRef]

3) Fujisawa $\mathrm{H}$, Suzuki $\mathrm{H}$, Nishiyama $\mathrm{T}$, et al.: Comparison of ankle planta flexor activity between double-leg heel raise and walking. J Phys Ther Sci, 2015, 27: 1523-1526. [Medline] [CrossRef]

4) Perry J, Burnfield JM: Gait Analysis; normal and pathological function. New Jersey: SLACK, 2010, pp 3-18, 51-82.

5) Schmidt RA, Lee TD: Motor control and learning; a behavioral emphasis. Illinois: Human Kinetics, 2011, pp 481-487.

6) Kim $\mathrm{MH}$, Yi CH, Weon JH, et al.: Effect of toe-spread-out exercise on hallux valgus angle and cross-sectional area of abductor hallucis muscle in subjects with hallux valgus. J Phys Ther Sci, 2015, 27: 1019-1022. [Medline] [CrossRef]

7) Kwon W, Jang H, Jun I: Comparison of supraspinatus cross-sectional areas according to shoulder abduction angles. J Phys Ther Sci, 2015, 27: 539 541. [Medline] [CrossRef]

8) Huang Q, Li D, Zhang J, et al.: Comparison of the efficacy of different long-term interventions on chronic low back pain using the cross-sectional area of the multifidus muscle and the thickness of the transversus abdominis muscle as evaluation indicators. J Phys Ther Sci, 2014, 26: 1851-1854. [Medline] [CrossRef]
9) Fukunaga T, Kubo K, Kawakami Y, et al.: In vivo behaviour of human muscle tendon during walking. Proc Biol Sci, 2001, 268: 229-233. [Medline] [CrossRef]

10) Kawakami $Y$, Muraoka $T$, Ito $S$, et al.: In vivo muscle fibre behaviour during counter-movement exercise in humans reveals a significant role for tendon elasticity. J Physiol, 2002, 540: 635-646. [Medline] [CrossRef]

11) Sugisaki N, Kanehisa H, Kawakami $Y$, et al.: Behavior of fascicle and tendinous tissue of medial gastrocnemius muscle during stretch-shortening cycle exercise of ankle joint. Int J Sport Health Sci, 2005, 3: 100-109. [CrossRef]

12) Hirayama $K$, Yanai $T$, Kanehisa $H$, et al.: Neural modulation of muscletendon control strategy after a single practice session. Med Sci Sports Exerc, 2012, 44: 1512-1518. [Medline] [CrossRef]

13) Galbraith RM, Lavallee ME: Medial tibial stress syndrome: conservative treatment options. Curr Rev Musculoskelet Med, 2009, 2: 127-133. [Medline] [CrossRef]

14) Woodley BL, Newsham-West RJ, Baxter GD: Chronic tendinopathy: effectiveness of eccentric exercise. Br J Sports Med, 2007, 41: 188-198, discussion 199. [Medline] [CrossRef]

15) Alfredson H, Cook J: A treatment algorithm for managing Achilles tendinopathy: new treatment options. Br J Sports Med, 2007, 41: 211-216. [Medline] [CrossRef]

16) Wasielewski NJ, Kotsko KM: Does eccentric exercise reduce pain and improve strength in physically active adults with symptomatic lower extremity tendinosis? A systematic review. J Athl Train, 2007, 42: 409-421. [Medline] 